\title{
Cross-Training to shared standards at the national cryoEM centers using "Merit Badges"
}

Christina Zimanyi ${ }^{1}$, Edward Eng ${ }^{2}$, Craig Yoshioka ${ }^{3}$, Sean Mulligan ${ }^{3}$, Claudia Lopez ${ }^{4}$, Corey Hecksel $^{5}$, Michael Schmid $^{6}$, Patrick Mitchell ${ }^{6}$, Lydia-Marie Joubert ${ }^{6}$, Peter Shen ${ }^{7}$, Janet Iwasa ${ }^{8}$, Grant Jensen ${ }^{9}$, Fred Sigworth $^{10}$, Brenda Gonzalez ${ }^{11}$, Yingjie Chen ${ }^{11}$, Wen Jiang ${ }^{11}$, Jiahui Dong ${ }^{11}$, Xingyu Jiang ${ }^{11}$ and Zongcheng $\mathrm{Chu}^{11}$

${ }^{1}$ New York Structural Biology Center, United States, ${ }^{2}$ New York Structural Biology Center, New York, New York, United States, ${ }^{3}$ Oregon Health and Science University, United States, ${ }^{4}$ OHSU Portland (OR), Portland, Oregon, United States, ${ }^{5}$ SLAC National Accelerator Laboratory, United States, ${ }^{6}$ SLAC National Accelerator Laboratory, Stanford University, United States, ${ }^{7}$ University of Utah, Salt lake City, Utah, United States, ${ }^{8}$ University of Utah, United States, ${ }^{9}$ Caltech, United States, ${ }^{10}$ Yale University School of Medicine, United States, ${ }^{11}$ Purdue University, United States

The mission of the Transformative High Resolution Cryo-Electron Microscopy (CryoEM) Program funded by the NIH Common Fund is to increase the national cryoEM research capacity. The program provides free-of-charge access to state-of-the-art instrumentation, technology, and expertise at cryoEM service centers. To expand the cryoEM workforce, the centers also provide hands-on intensive crosstraining programs to train labs without prior experience to become self-sufficient at cryoEM techniques. Also supporting the goal of expanding the cryoEM workforce, the program has funded four groups to develop instructional materials and educational tools that can be used independently, particularly with online and self-paced components to teach CryoEM techniques, application, theory, and analysis.

The three cryoEM service centers (National Center for CryoEM Access and Training, the Pacific Northwest CryoEM Center, \& the Stanford SLAC CryoEM Center) and the four curriculum development programs (CryoEM 101, Getting Started in CryoEM, CryoVR, \& Principles of CryoEM) are independent but share a common training mission. As demand for intensive hands-on training is higher than current capacity at any of the centers, we hope to leverage the work being done by each group to develop best practices for efficient cross-training.

Towards this aim, we have established a working group with representation from each of the service centers and the curriculum development teams to create a system of "Merit Badges", a certification of competency in the independent use of a specific instrument or workflow that will be cross-honored at each of the national service centers. Merit badges are modular and organized into units comprising a single step of the cryoEM workflow. Initially, they will be awarded to users in three main skill areas: 1) Sample preparation, 2) Microscope operations and 3) Data processing. A modular collection of badges allows for easily customized training plans for researchers with different needs. Once badges in these initial areas are established, the program will expand to allow differentiated instruction and certification for varying types of practitioners, including those that are focused on theory and data analysis. 
A merit badge has two key components: 1) demonstration of essential foundational knowledge with a simple test and 2) demonstration of practical proficiency by in-person evaluation from center staff. The main objectives of the working group are to identify the minimal essential theoretical knowledge to test for each practical skill, to organize already developed instructional content to teach that knowledge (and avoid the need to generate more training materials) and to agree to a standard of evaluation shared between the centers.

The incentive for trainees to obtain a merit badge is that it certifies them as qualified to use a particular instrument or workflow independently at any national service center after a brief orientation of center specific procedures and safety rules. The incentive for the centers is to reduce staff time needed for training and to foster collaboration on best practices.

In this talk, I will introduce the merit badge concept, update on our progress, and propose potential additional applications where we believe this framework may be useful for establishing current best practices in the cryoEM community without discouraging or hindering future experimentation, innovation, and development of any of these methods. 